\title{
Fumonisins: toxicology, emerging issues, and prospects for control and detoxification
}

\author{
Kenneth A.Voss ${ }^{* 1}$, Ronald T. RILEY ${ }^{* 1}$, Janee B. GelineaU-VAN WAES ${ }^{* 2}$ and \\ Charles W. BACON ${ }^{* 1}$
}

${ }^{* 1}$ Toxicology \& Mycotoxin Research Unit, USDA Agricultural Research Service,

(P. O. Box 5677 Athens, Georgia, USA)

${ }^{* 2}$ Department of Genetics, Cell Biology, and Anatomy, Munroe-Meyer Institute,University of Nebraska Medical Center,

(Omaha, Nebraska, USA)

\section{Introduction}

Fumonisins are produced by Fusarium verticillioides (formerly $F$. moniliforme Sheldon) and related Fusarium species ${ }^{1,2}$. They occur in corn and in corn-based products worldwide and both fumonisins and fumonisin-producing fungi cause toxic effects in animals ${ }^{1-3)}$ (Table 1). Their risk to humans is unclear ${ }^{1)}$ but both are implicated as factors for esophageal cancer ${ }^{1)}$ and, more recently, neural tube defects ${ }^{1,4}$. While much has been learned about fumonisins, research to further characterize their toxicity, their mode of action, as well as to determine their relevance to human health continue. Recent research on some issues of emerging importance is reviewed below.

\section{Fumonisin toxicology and mechanism of action}

The toxicology and mode of action of fumonisins have been extensively reviewed elsewhere and comprehensive reference lists of original research reports are available in these publications ${ }^{1-3)}$ (Table 1). Fumonisins' mechanism of action is multifaceted but evidence indicates that inhibition of ceramide synthase (fumonisins are structurally similar to the enzyme's sphingoid base substrates sphinganine $(\mathrm{Sa})$ and sphingosine (So)) is the initial mechanistic event ${ }^{5}$. Consequently, sphingolipid metabolism is disrupted and $\mathrm{Sa}$ and So accumulate in cells and tissues, cell and tissue complex sphingolipids are depleted, and So (Sa)-1-phosphates and other sphingoid base metabolites accumulate. Consequently, critical sphingolipid-mediated cell signaling pathways or sphingolipid-dependent physiological functions are disrupted. For example, So-1-phosphate binds to G protein-linked Edg receptors and causes vasoconstriction of rat mesenteric and renal microvessels in vitro ${ }^{6}$ and in vivo ${ }^{7}$. It is therefore possible that 
accumulated So-1-phosphate in the kidney of fumonisin-exposed rats might reduce blood flow and exacerbate apoptosis. Likewise, accumulated sphingoid bases in the serum or tissues might be the mechanistic trigger for cardiac insufficiency and, ultimately, pulmonary edema in swine ${ }^{8)}$.

\section{Reproductive toxicity revisited}

In previous studies, $F$. verticillioides and $\mathrm{FB}_{1}$ were not teratogenic to rodents and rabbits and embryotoxicity, when present, occurred only at maternally toxic doses ${ }^{1,3)}$. However, in several reports, decreased fetal birth weight, and/or increased fetal resorption were found following maternal $\mathrm{FB}_{1}$ exposure. Ex utero exposure to $\mathrm{FB}_{1}$ caused developmental defects in rat embryos (including exencephaly) ${ }^{1.3)}$ but, because ${ }^{14} \mathrm{C}$ $\mathrm{FB}_{1}$ did not cross the placenta of pregnant rats (on Gestation Day 15) and increased Sa or $\mathrm{Sa} / \mathrm{So}$ (biomarkers of fumonisin exposure ${ }^{1.5)}$ ) was not found in fetuses of dams (rats, mice and rabbits) given $\mathrm{FB}_{1}$, it was concluded that the placenta was an effective barrier to in utero exposure ${ }^{3)}$.

Fumonisins' potential as a risk factor for birth defects, specifically neural tube defects (cranial and spinal cord abnormalities ranging from spina bifida occulta to exencephaly or craniorhachischisis), has again become a concern. A spike in the incidence of neural tube defects within the Hispanic population near Brownsville, TX in 1990-91, a time when fumonisin levels in locally grown corn were coincidentally high, suggested a possible connection ${ }^{4}$. In this regard, it is interesting to note that neural tube defect incidences are also high in regions of southern Africa, including the Transkei, and elsewhere where fumonisin exposure is likely to be high ${ }^{1)}$.

Stevens and Tang ${ }^{9)}$ found that $\mathrm{FB}_{1}$ inhibited carrier-mediated folate transport in Caco-2 cells. Folate deficiency during a critical phase of early pregnancy is associated with neural tube defects and the high-affinity placental folate transporter (folbp 1 in mice and folate receptor alpha in man) is a sphingolipid-associated GPI-anchored protein. It was therefore hypothesized that fumonisins indirectly contribute to neural tube defects by depleting membrane sphingolipids that are critical for folate receptor function". Consistent with this hypothesis are the findings of Sadler et al. ${ }^{10}$, that showed folinic acid supplementation could significantly reduce the incidence of $\mathrm{FB}_{1}$-induced neural tube defects in murine embryo culture.

Gelineau-van Waes and colleagues have studied maternal exposure and neural tube defects using the inbred LMBc mouse as an in vivo model ${ }^{11}$. In summary, they found dose-dependent increases in neural tube defects (exencephaly) in the litters of dams given intraperitoneal doses of $\mathrm{FB}_{1}$ on gestation days 7.5 and 8.5 ; the incidence of affected fetuses ranged from 2 percent (dose $=5 \mathrm{mg} \mathrm{FB} / \mathrm{kg}$ maternal $\mathrm{BW}$ ) to 79 percent $(20 \mathrm{mg} / \mathrm{kg})$. Increased $\mathrm{Sa}$ concentration and $\mathrm{Sa} / \mathrm{So}$ were found not only in the livers and 
Table 1. A summary of selected toxic effects and diseases caused by fumonisins. 1

\begin{tabular}{|c|c|}
\hline Species & Description and Comments \\
\hline Horse & $\begin{array}{l}\text { Equine Leukoencephalomalacia (ELEM): ELEM is characterized by hemorrhagic \& } \\
\text { liquifactive lesions of white matter. Its clinical onset and course are rapid and its } \\
\text { neurological signs include lethargy, weakness, recumbency and facial paralysis. } \\
\text { ELEM is unique to Equidae. Liver involvement similar to that in other species also } \\
\text { occurs. }\end{array}$ \\
\hline
\end{tabular}

Swine Porcine Pulmonary Edema (PPE): PPE is characterized by rapidly developing accumulations of fluid in alveolar membranes and spaces. Animals subsequently die of respiratory failure ${ }^{8)}$. PPE might be the result of compromised cardiac contractility. PPE is unique to swine, but the liver is also a target organ.

Rat Liver and kidney are the main target organs. Apoptosis is an early finding and with prolonged exposure, necrosis, regenerative and other lesions develop. Serum chemistry findings support the pathology. FB1 promotes enzyme (GGT and GST) positive liver foci and is a liver and kidney carcinogen in rats $(50 \mathrm{mg} / \mathrm{kg}$ in diet), although differences in target organs occurr by sex (male vs. female F344/Nnctr rats) and rat strain (F344/Nnctr males vs BD IX males). Other effects including thymic apoptosis, inhibition or enhancement of immune or host-resistance responses, changes in hepatic gene expression, or indications of oxidative injury have been reported.

Mouse The liver is the primary target organ in mice. Serum chemistry and pathology findings are consistent with those found in rats and, as in rats, apoptosis and mitosis/regeneration likely play a key role in pathogenesis. The kidney is also a target, but the mouse is not as susceptible as Sprague-Dawley and F344 rats to fumonisin nephrotoxicity. FB 1 ( $50 \mathrm{mg} / \mathrm{kg}$ in the diet) was hepatocarcinogenic to female B6C3F1 mice. Gene knockout strains have been useful for studying how cytokines and various receptors mediate fumonisin toxigenesis.

Rabbit The liver and kidney are target organs. Lesions and other findings are similar to those found in rodents. Pregnant rabbits are especially sensitive to $\mathrm{FB}_{1}$.

Nonhuman The liver is a target organ based on pathology (apoptosis, perilobular fibrosis, Primates nodular hyperplasia and other lesions in liver) and serum chemistry endpoints in vervet monkeys chronically fed $F$. verticillioides culture material.

*1 Extensive citation lists of original research reports by the numerous groups that contributed information to this table is given in the review of fumonisins published by the FAO/WHO Joint Expert Committee on Food Additives ${ }^{1)}$ and elsewhere ${ }^{3)}$. 
kidneys of the treated dams, but also in their placentas embryos and biodistribution studies confirmed that ${ }^{14} \mathrm{C}-\mathrm{FB}_{1}$ crossed the placenta on gestation day 10.5. Why these findings contrast with earlier results ${ }^{3}$ is not known, however, the LMBc studies were done much earlier in gestation, at a time when the placenta is less developed and less likely to provide an effective barrier against mycotoxin exposure. The $\mathrm{FB}_{1}$ dose, the route and timing of dosing, or species/strain-related differences in placental physiology, sphingolipid metabolism, and/or genetic susceptibility to neural tube defects are also possible contributing factors.

In any event, folate plays a role in the $\mathrm{LMBc}$ mouse model. Fetal folate uptake was decreased by $\mathrm{FB}_{1}$ and daily oral administration of folinic acid to the high-dose dams $(n=10)$ beginning on the first day of gestation reduced $\mathrm{FB}_{1}$-induced neural tube defect incidence from 79 to 43 percent. Administering the complex sphingolipid ganglioside $\mathrm{G}_{\mathrm{M} 1}$ on gestation days $6.5-9.5(10 \mathrm{mg} / \mathrm{kg} / \mathrm{day}$ ip) more effectively counteracted neural tube defect induction by $\mathrm{FB}_{1}$, as the incidence of neural tube defects in the fetuses of dams $(n=11)$ treated with the high-dose of $F_{1}$ plus $G_{M 1}$ was only five percent. Immunohistochemical expression of the folate receptor and ganglioside $G_{M 1}$ in the yolk sac membrane and neuroepithelium of the embryos diminished following $\mathrm{FB}_{1}$ exposure, also suggesting a connection between sphingolipid depletion and decreased folate transport. These investigations have established the LMBc mouse as a useful model for investigating how fumonisins, sphingolipids, and folate interact to affect neural tube closure. However, given the species/strain-dependent differences in response to fumonisins that occur, more studies in LMBc mice and other mouse strains and species are needed to place these findings in perspective. These include: determining doseresponse and the no observed effect level in $\mathrm{LMBc}$ dams exposed to $\mathrm{FB}_{1}$ via the diet; determining the influence of maternal toxicity on litter neural tube defect incidence; investigating transplacental transport of folate and fumonisins as a function of time (gestation day); establishing if folate transport in LMBc dams is uniquely sensitive to $\mathrm{FB}_{1}$; defining more fully the molecular and pathophysiological mechanisms underlying induction of neural tube defects in LMBc mice and, determining the extent to which these mechanistic findings apply to other mouse strains and species.

\section{Cooking and "hidden" fumonisins}

There is evidence that "hidden" fumonisins (matrix-bound forms not detected using "routine" extraction and analysis methods) are present in some foods (Table 2). Fumonisin-matrix interactions and reaction products in foods are not well characterized. Several have been detected (Table 2) and others are possible. When extracted (using a "routine" method) cornflake residues were re-extracted with sodium dodecyl sulfate and the second extract then base hydrolysed, a significant amount (2.6 times the amount 
Table 2. Examples of Fumonisin Reaction Products and their Potential Biological Activity. Refer to the cited reports for experimental details.

Product Formation, Toxicology Data, and Analysis Methods

Hydrolyzed HFBs are formed by base hydrolysis of fumonisins during nixtamalization (cooking in Fumonisins ${ }^{1}$. alkaline water) of corn to make masa, the basic ingredient of tortillas. HFBs occur in (HFBs) masa products, although usually in lesser amounts than fumonisins. In vitro and in vivo bioassay results are variable but the weight of evidence suggests HFBs are no more and likely less toxic than fumonisins. HFBs can be measured by HPLC or mass spectroscopy ${ }^{13-15)}$. HFBs substitute for sphingoid bases as substrates for ceramide synthase ${ }^{29)}$.

Maillard N-(Carboxymethyl)- and $N$-(deoxy-D-fructos-1-yl)-fumonisins form when reaction fumonisins are heated in the presence of reducing sugars such as glucose. The extent products1. to which these products occur in foods is not well studied, but significant $\mathrm{N}$ (carboxymethyl)- or $N$-(1-deoxy-D-fructos-1-yl)-fumonisins were not found in masa and tortilla chips. $N$-(Carboxymethyl)-FB। was not toxic to mice ${ }^{25)}$ and toxicity to rats of $\mathrm{FB}_{1}$-glucose reaction product(s) was reduced compared to $\mathrm{FB}_{1}{ }^{24)}$ possibly due to limited bioavailability of the fumonisin-sugar compound, lack of a free amino function on the reaction product, or both. These compounds are measured using mass spectroscopy or other methods not dependent on derivatization of the amino group ${ }^{16,27)}$.

3-keto 3-keto $\mathrm{FB}_{1}$ was formed by ozonation (a candidate detoxification method) of an fumonisins aqueous $\mathrm{FB}_{1}$ solution. 3-keto $\mathrm{FB}_{1}$ retains biological activity ${ }^{28)}$ but its toxicity has not been tested in vivo in mammalian or avian species. 3-keto $\mathrm{FB}_{1}$ can be quantified by routine HPLC or mass spectrometry methods ${ }^{23)}$.

Deaminated Deaminated $\mathrm{FB}_{1}$ is formed by reaction of $\mathrm{FB}_{1}$ with $\mathrm{NaNO}_{2}{ }^{28}$. Deaminated $\mathrm{FB}_{1}$ was fumonisins not toxic to Hydra and did not inhibit ceramide synthase in vitro. Bioavailability and toxicity has not been tested in mammals. The deaminated product can be quantified using mass spectrometry but the loss of the amino group precludes detection of the deamination product using "routine" HPLC methods for fumonisins.

Fumonisin- Heating FB1, with sucrose, methyl $\alpha$-D-glucosylpyranoside (a starch model), or starch \& $\quad N$ - $\alpha$-acetyl-L-lysine methyl ester and BOC-L-cysteine methyl ester (protein protein surrogates), yielded conjugation products. In contrast to $\mathrm{FB}_{1}$-reducing sugar products, conjugates the sugar (or amino acid) bound to the tricarballylic acid function of $F_{1}$. It is possible that conjugated fumonisins could become bioavailable by hydrolysis of the conjugated product during digestion. Analysis is difficult but can be indirectly accomplished by HPLC or mass spectrometric analysis of hydrolyzed fumonisins after base hydrolysis of the food or grain sample ${ }^{16)}$. It is possible that these might contribute to "hidden" fumonisins in some foods ${ }^{12}$.

${ }^{* 1}$ Fumonisin reaction products have been detected in foodstuffs. 
measured initially) of "hidden" fumonisins was liberated ${ }^{12}$. The toxicological importance of these "hidden" fumonisins in foods depends upon their concentration, bioavailability, and inherent toxicity.

Cooking variably reduces measurable fumonisins in foods and consequently might reduce toxicity. For example, cooking in water significantly reduced both fumonisin concentration (ca. $90 \%$ ) and in vivo toxicity to rats of $F$. verticillioides molded corn ${ }^{13)}$. Nixtamalization (cooking/steeping in alkaline water) extracts fumonisins from corn and reduces their amounts in masa and tortilla products by as much as 80 percent $^{14,15}$. The fate of the remaining fumonisins, originally present in the corn, was not determined in these experiments but only trace amounts of Maillard reaction products (see explanation in Table 2) were detected in the masa ${ }^{15}$. In addition to Maillard reaction products, fumonisin-starch or -protein conjugates can be formed by reactions involving fumonisin's tricarballylic acid groups ${ }^{16)}$. This has potentially important toxicological implications because these compounds are not readily detected by routine analytical procedures and also because they might break down in the intestines, liberating free fumonisi ${ }^{16}$. The possible formation of unknown toxic fumonisin reaction products during cooking must also be considered.

To circumvent any analytical uncertainty, the effect of cooking on fumonisin toxicity was evaluated using an in vivo bioassay ${ }^{17}$. Briefly, pan-fried corncakes, deepfried fritters, and baked cornbread were made from cornmeal that was spiked with $F$. verticillioides culture material. The cooked products, uncooked spiked cornmeal, or uncontaminated cornmeal (high dose only) were fed to rats at a low ( $2 \% \mathrm{w} / \mathrm{w}$ mixed into rodent chow) or high $(20 \% \mathrm{w} / \mathrm{w})$ doses for two weeks. The toxic effects induced by the cooked products and the uncooked spiked cornmeal, as determined by microscopic evaluation of liver and kidneys, were indistinguishable and typical of those caused by fumonisins. Measured fumonisin ( $\mathrm{FB}_{1}+\mathrm{FB}_{2}$; HPLC analysis) concentrations of the highdose pan-fried corncake diet $(92 \mathrm{mg} / \mathrm{kg})$ were 30 percent less than that of the high-dose uncooked spiked cornmeal diet $(131 \mathrm{mg} / \mathrm{kg})$ but baking and deep-frying had essentially no effect on fumonisin concentration (120-132 mg/kg in the products). It could not be concluded that any "hidden" or unknown fumonisins formed during pan-frying because, at the dose levels used in the study, a $30 \%$ reduction in fumonisin concentration is unlikely to affect dose-response. Importantly, however, the results indicated that baking and frying did not enhance toxicity. More investigations are needed to further elucidate how various cooking methods and recipes affect chemical fate and toxicity of fumonisins, especially in foods prepared from naturally contaminated corn having low $(<5 \mathrm{mg} / \mathrm{kg}$ ) fumonisin concentrations, and to optimize cooking conditions to reduce fumonisins in food products. 


\section{Prospects for detoxification}

Strategies for reducing fumonisins in foods include controlling the growth of mycotoxigenic fungi in the field. In this regard, breeding programs to produce hybrid plants that do not accumulate fumonisins ${ }^{18)}$ or competitive exclusion using fumonisin non-producing fungi ${ }^{19)}$ or nontoxic bacteria ${ }^{20)}$ might be effective. Developing transgenic plants that resist insect damage caused by corn borers and earworms ${ }^{21}$ might also prove to be useful, however, none of these approaches have yet been developed into a management program proven to reduce $F$. verticillioides or fumonisins in corn under field conditions.

Chemical and physical treatments for detoxifying fumonisins have produced variable success (Table 2). Ammoniation using a procedure effective for reducing aflatoxin in corn was ineffective against fumonisins ${ }^{22}$. Ozone treatment ${ }^{23)}$ was likewise ineffective against $\mathrm{FB}_{1}$ as the reaction product, a 3-keto $\mathrm{FB}_{1}$, was biologically active. On the other hand, $\mathrm{FB}_{1}$-reducing sugar reaction (Maillard reaction) products were not toxic to rats ${ }^{24)}$ or mice ${ }^{25)}$. The addition of glucose to cornmeal or grits likewise decreased the amount of $\mathrm{FB}_{1}$ measured in baked and extruded foods ${ }^{26)}$ but the reaction products have not been characterized. Significant amounts of $N$-(carboxymethyl)-FB $\mathrm{FB}_{1}$ were not detected and the presence or absence of $N$-(deoxy-D-fructos-1-yl)-FB ${ }_{1}$ or fumonisin-sugar (protein) conjugates ${ }^{16)}$ was not reported.

\section{Summary}

Much has been learned about fumonisins since their discovery and investigations to further characterize their toxicity and mode of action continue. A recently developed mouse model for neural tube defects in fetuses of fumonisin-treated dams will be a valuable in vivo tool for elucidating the mechanistic relationships between fumonisin exposure, folate transport, and neural tube defects in this mouse model and for determining the relevance of the mouse model for humans. Determining how cooking and food processing affect fumonisins in food matrices and, using an approach combining bioassays and chemical analyses, their toxicity is another emerging research initiative. Ongoing research in these and other areas will contribute to better understanding of fumonisins and to the implementation of science-based mycotoxin management strategies.

Key words : fumonisins, toxicity, folate, review

\section{References}

1 ) Joint FAO/WHO Expert Committee on Food Additives (JECFA): Safety Evaluation of Certain 
Mycotoxins in Foods. (2001) World Health Organization. Geneva.

2 ) Haschek, W.M., Voss, K.A., Beasley, V.R.: Handbook of Toxicologic Pathology, 2nd Edition, (eds. Haschek, W.M., Rousseaux. C.G., Wallig, M.A.) pp 645-699 (2002) Academic Press, San Diego.

3 ) Voss, K.A., Riley, R.T., Norred, W.P., Bacon, C.W., Meredith, F.I., Howard, P.C., Plattner, R.D., Collins, F.T.X., Hansen, D.K., Porter, J.K.: Environ. Health Perspect., 109 (Suppl. 2), 259266 (2001)

4 ) Hendricks, K.: Epidemiology., 10, 198-200 (1999)

5 ) Riley, R.T., Enongene, E.N., Voss, K.A., Norred, W.P., Meredith, F.I., Sharma, R.P., Spitsbergen, J., Williams, D.E., Carlson, D.B., Merrill, A.H., Jr.: Environ. Health Perspect., 109 (Suppl. 2), 301-308 (2001)

6 ) Bischoff, A., Czyborra, P., Fetscher, C., zu Heringdorf, D.M., Jakobs, K.H., Michel, M.C.: Brit. J. Pharmacol., 130, 1871-1877 (2000)

7 ) Bischoff, A., Czyborra, P., zu Heringdorf, D.M., Jakobs, K.H., Michel, M.C.: Brit. J. Pharmacol., 130, 1878-1883 (2000)

8 ) Constable, P.D.; Smith, G.W.; Rottinghaus, G.E.; Haschek, W.M.: Toxicol. Appl. Pharmacol., 162, 151-160 (2000)

9 ) Stevens, V.L., Tang, J.: J. Biol. Chem., 272, 18020-18025 (1997)

10) Sadler, T.W., Merrill, A.H., Jr., Stevens, V.L., Sullards, M.C., Wang, E., Wang, P.: Teratology., 66, 169-176 (2002)

11) Starr, L., Riley R., Voss, K., Maddox, J., Bennett, G., Gelineau-van Waes, J.: Toxicological Sci. (The Toxicologist) 1S, 171 (2003)

12) Kim, E.-K., Scott, P.M., Lau, B.P.-Y.: Food Addit. Contam., 20, 161-169 (2003)

13) Voss, K.A., Riley, R.T., Bacon, C.W., Meredith, F.I., Norred, W.P.: Environ. Toxicol. Pharmacol., 5, 101-104 (1998)

14) Dombrink-Kurtzman, M.A., Dvorak, T.J., Barron, M.E., Rooney, L.W.: J. Agric. Food Chem., 48, 5781-5786 (2000)

15) Voss, KA., Poling, S.M., Meredith, F.I., Bacon, C.W., Saunders, D.S.: J. Agric. Food Chem., 49, 3120-3126 (2001)

16) Seefelder, W., Knecht, A., Humpf, H.-U.: J. Agric. Food Chem., 51, 5567-5573 (2003)

17) Voss, K.A., Meredith, F.I., Bacon, C.W.: J. Agric. Food Chem., 57, 5546-5551 (2003)

18) Duvick, J.: Environ. Health Perspect., 109 (Suppl. 2), 337-342 (2001)

19) Desjardins, A.E., Plattner, R.D.: J. Agric. Food Chem., 48, 5773-5780 (2000)

20) Bacon, C.W., Yates, I.E., Hinton, D.M., Meredith, F.I.: Environ. Health Perspect., 109 (Suppl. 2), 325-332 (2001)

21) Munkvold, G.P., Hellmich, R.L., Rice, G.L.: Plant Dis., 83, 130-138 (1999)

22) Norred, W.P., Voss, K.A., Bacon, C.W., Riley, R.T.: Fd Chem Toxicol., 29, 815-819 (1991)

23) McKenzie, K.S., Sarr, A.B., Mayura, K., Baily, R.H., Miller, D.R., Rogers, T.D., Norred, W.P., Voss, K.A., Plattner, R.D., Kubena, L.F., Phillips, T.D.: Fd Chem. Toxicol., 35, 807-820 (1996)

24) Liu H.; Lu, Y.; Haynes, J.S.; Cunnick, J.E.; Murphy, P.; Hendrich, S.: J. Agric. Food Chem., 49, 4113-4121 (2001)

25) Howard P.C., Couch, L.H., Patton, R.E., Eppley, R.M., Doerge, D.R., Churchwell, M.I., Matilde Marques, M., Okerberg, C.V.: Toxicol. Appl. Pharmacol., 185, 153-165 (2002)

26) Castelo, M.M., Jackson, L.S., Hanna, M.A., Reynolds, B.H., Bullerman, L.B.: J. Food Sci., 66, 416-421 (2001) 